\title{
REEF3D::NSEWAVE, A Three-Dimensional Non-Hydrostatic Wave Model on a Fixed Grid
}

\author{
Hans Bihs, Norwegian University of Science and Technology NTNU Trondheim, Norway, hans.bihs@ntnu.no \\ Kristina Heveling, Karlsruhe Institute of Technology KIT, Germany, kristinah@web.de \\ Arun Kamath, Norwegian University of Science and Technology NTNU Trondheim, Norway, arun.kamath@ntnu.no
}

\section{INTRODUCTION}

For coastal engineering problems, wave modeling is required for various spatial scales. In recent years, the development of high-resolution Computational Fluid Dynamics (CFD) based numerical wave tanks (NWT) has gained a lot of attention. Here, the Navier-Stokes equations are solved together with a two-phase interface capturing algorithm for the calculation of the free surface location. The interface capturing treatment of the free water surface is performed on fixed grids, allowing for the simulation of complex wave phenomena such as breaking waves. The CFD-based NWT are preferably used for nearfield problems, such as wave-structure interaction. For larger spatial scales, the computational cost becomes rather expensive. In the current paper, the three-dimensional open-source hydrodynamics model REEF3D is extended from a CFD-based NWT to a nonhydrostatic wave model, suitable for economic large scale computation of waves.

\section{NUMERICAL MODEL}

The governing equations solved by REEF3D (Bihs et al., 2016) are the Navier-Stokes equations for the flow velocities. The model discretizes the velocities with a high-order WENO scheme in a conservative finite differences framework on a staggered grid for tight pressure-velocity coupling. The pressure is treated with the projection method, and the resulting Poisson equation is solved with a geometric multigrid preconditioned conjugated gradient solver from the HYPRE library. The numerical model is fully parallelized based upon the domain decomposition strategy, employing MPI for node-to-node communication. When used as a CFD-based NWT (REEF3D::CFD), the free surface is captured with the level set method. The level set function is propagated in time and space with a convection equation, which can be discretized with highorder finite differences. This approach sets certain requirements for the mesh resolution around the interface.

In the present study, REEF3D::NSEWAVE is introduced. This module follows the general numerical framework of REEF3D::CFD. The major difference is the treatment of the free surface: Instead of solving the convection equation for the level set function, the dynamic free surface is calculated based on the divergence of the depth-integrated continuity equation. This interface tracking method can be typically found in $\sigma$-coordinate type wave models, where the free surface is represented by a moving mesh. In the current model, the mesh is not moved. Instead, the location of the free surface is still given with the implicit geometric description of a signed distance function. As a result, it inherits the speed, robustness and accuracy of REEF3D::CFD. The mesh requirements around the free surface for the convection of the level set function are not present in REEF3D::NSEWAVE, which allows for the use of much coarser grids and larger time steps. capabilities to be employed on relatively coarser grids, wave propagation in an empty $2 \mathrm{D}$ tank is modeled. The wave tank is $20 \mathrm{~m}$ long and $1 \mathrm{~m}$ high. The water depth is $0.5 \mathrm{~m}$. The wave height is $0.02 \mathrm{~m}$ and the wave length is $2 \mathrm{~m}$. Waves are generated and absorbed with the relaxation method. Figure 1 shows the comparison between REEF3D::NSEWAVE and REEF3D::CFD on a mesh with the relatively coarse uniform mesh width $\mathrm{dx}=0.1 \mathrm{~m}$. At this resolution, the waves in the CFD-based NWT experience visible amplitude damping. On the other hand, REEF3D::NSEWAVE does not suffer from this problem, even though the water depth is only covered by five cells.

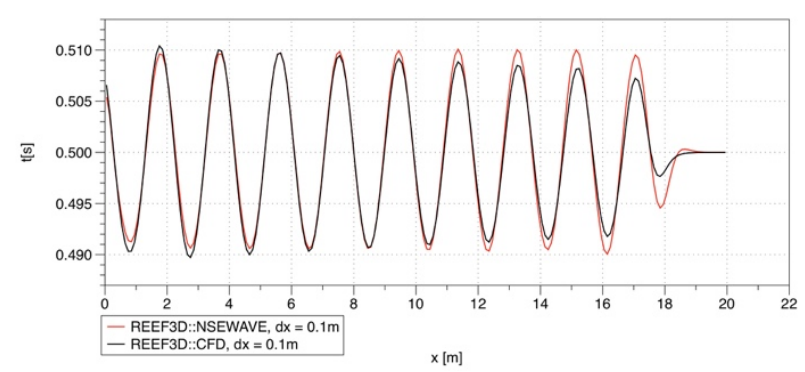

Figure 1 - Comparison of REEF3D::NSEWAVE and REEF3D::CFD for wave propagation in an empty wave tank.

When compared to experimental data from Beji and Battjes, the numerical results are shown for the last wave gage at $x=21 \mathrm{~m}$. At this point, shoaling and decomposition of the waves have occurred over the slopes of the submerged bar and the complex free surface patter is challenging to model.

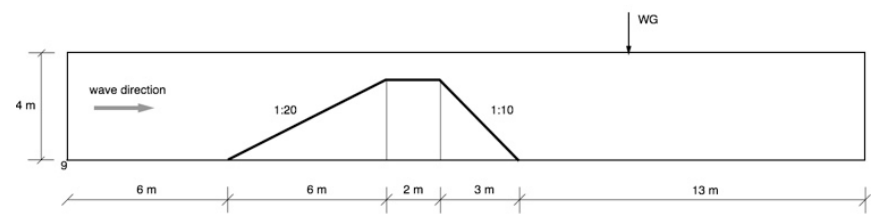

Figure 2 - Experimental setup for submerged bar (Beji and Battjes, 1993).

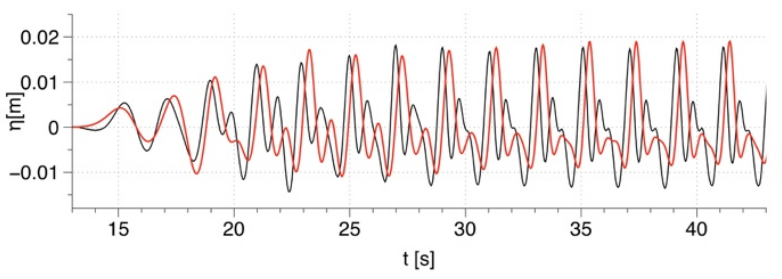

Figure 3 - Numerical results for $d x=0.05 m$ (red line) compared with experimental data (black line) for submerged bar.

For the mesh at $\mathrm{dx}=0.05 \mathrm{~m}$, a phase shift can be clearly observed, indicating a too coarse mesh. At the same time, the primary peak is only slightly underestimated. At 
the increased resolution of $\mathrm{dx}=0.02 \mathrm{~m}$, the numerical results improve significantly (see Figure 4). For the mesh width of $d x=0.01 \mathrm{~m}$ in Figure 5 , very good results are achieved.

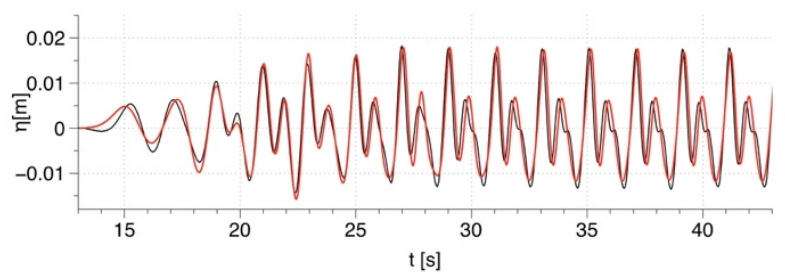

Figure 4 - Numerical results for $\mathrm{dx}=0.02 \mathrm{~m}$ (red line) compared with experimental data (black line) for submerged bar.

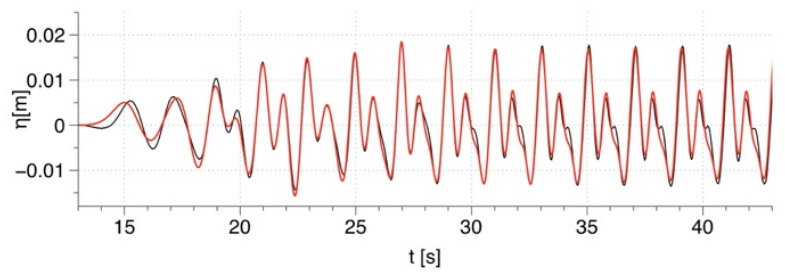

Figure 5 - Numerical results for $d x=0.02 \mathrm{~m}$ (red line) compared with experimental data (black line) for submerged bar.

A real-word application of REEF3D::NSEWAVE is the prediction of wave propagation and transformation around the Norwegian harbor of Andenes which is located at the northernmost tip of the island Andøya in the Vesterålen archipelago (see Figure 6).

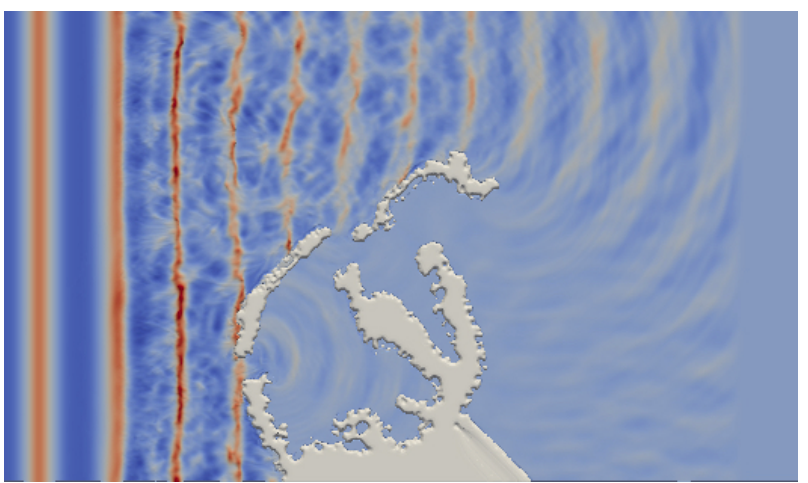

Figure 6 - Wave Propagation around Andenes Harbour

\section{CONCLUSIONS}

A new interface tracking method has been implemented in REEF3D::NSEWAVE, allowing for the accurate simulation of waves on relatively coarser meshes. This makes the model well equipped for the solution of larger scale wave problems, compared to CFD-based NWTs.

\section{REFERENCES}

Beji, Battjes (1993), Experimental investigation of wave propagation over a bar, Coastal Engineering, Vol. 19, pp.151162.

Bihs, Kamath, Alagan Chella, Aggarwal, Arntsen (2016): A new level set numerical wave tank with improved density interpolation for complex wave hydrodynamics, Computers and Fluids, vol. 140, pp. 191-208. 